\title{
Structure factor and electronic structure of compressed liquid rubidium
}

\author{
Junzo Chihara \\ Advanced Photon Research Center, Japan Atomic Energy Research Institute, Tokai, Ibaraki 319-11, Japan \\ Gerhard Kahl \\ Institut für Theoretische Physik and CMS, Technische Universität Wien, Wiedner Hauptstraße 8-10, A-1040 Wien, Austria
}

(Received 13 January 1998; revised manuscript received 10 March 1998)

\begin{abstract}
We have applied the quantal hypernetted-chain equations in combination with the Rosenfeld bridge functional to calculate the atomic and the electronic structure of compressed liquid rubidium under high pressure $(0.2,2.5,3.9$, and $6.1 \mathrm{GPa})$; the calculated structure factors are in reasonable agreement with experimental results measured by Tsuji et al. along the melting curve as a whole. It is found that the effective ion-ion interaction is practically unchanged with respect to the potential at room pressure under these high pressures. All structure factors calculated for this pressure-variation coincide almost into a single curve if wave numbers are scaled in units of the Wigner-Seitz radius $a$ although no corresponding scaling feature is observed in the effective ion-ion interaction. This scaling property of the structure factors signifies that the compression in liquid rubidium is uniform with increasing pressure; in absolute $Q$ values this means that the first peak position $\left(Q_{1}\right)$ of the structure factor increases proportionally to $V^{-1 / 3}$ ( $V$ being the specific volume per ion), as was experimentally observed by Tsuji et al. Obviously, this scaling property comes from a specific feature characteristic for effective ion-ion potentials of alkali liquids. We have examined and confirmed this feature for the case of a liquid-lithium potential: starting from the liquid-lithium potential at room pressure we can easily find two sets of densities and temperatures for which the structure factors become practically identical, when scaling $Q$ in units of $a$. [S0163-1829(98)00533-5]
\end{abstract}

\section{INTRODUCTION}

Based on density-functional (DF) theory, we have derived in previous work a set of integral equations which allows us to calculate the ion-ion and the electron-ion correlations in a liquid metal or a plasma, consistent with the bound-electron structure of the ion using only the atomic number $Z_{\mathrm{A}}$ as input; these integral equations are named the quantal hypernetted-chain (QHNC) equations, ${ }^{1,2}$ which are derived from the exact expressions for the ion-ion and electron-ion radial distribution functions (RDF) in an electron-ion mixture. Up to now, we have applied this approach to liquid metallic hydrogen, ${ }^{1}$ lithium, ${ }^{3}$ sodium, ${ }^{4}$ potassium, ${ }^{5}$ and aluminum $^{6}$ obtaining ion-ion structure factors in excellent agreement with experiments. Since a liquid metal can be considered as a very special type of a high-density plasma, we can expect from the successful application of the QHNC method to liquid metals that this approach is able to provide accurate results for a plasma. In such a system, both the ionic valency $Z_{\mathrm{I}}$ and the electron-ion interaction $v_{\mathrm{eI}}(r)$ may vary over a wide range as temperature and density are changed. Our method is in particular suited to treat a plasma, since it is able to calculate the ionization $Z_{\mathrm{I}}$ and the electron-ion interaction in a self-consistent manner using the atomic number of the system as the only input data. In a similar spirit, Perrot $^{7}$ has proposed the neutral-pseudoatom (NPA) theory to give an $a b$ initio calculation of effective ion-ion potentials for plasmas. González et al. have successfully applied this theory to alkali liquids ${ }^{8}$ and alkaline-earth liquids. ${ }^{9}$ The NPA method can be derived from the QHNC theory with use of additional approximations; ${ }^{10}$ the fundamental one is that the ion-ion correlation is approximated by the step function in the determination of the pseudopotential. This model was called in our approach the jellium-vacancy model ${ }^{4}$ and was used to obtain an accurate initial guess for effective ion-ion interactions in the iteration solving the QHNC equation.

Recently, we have extended this formalism and have performed a first-principles molecular dynamics (MD) simulation based on the QHNC theory for alkali metals near the triple point: in this study those small deviations which were still observed between experimental results and QHNC data for the structure factor disappeared completely. ${ }^{11}$ Nevertheless, the calculation of the ion-ion RDF in an MD simulation is rather time consuming. Recently, Rosenfeld ${ }^{12}$ has proposed a bridge functional for hard spheres; its construction requires only fundamental measures of the hard spheres. In combination with the MHNC approach-by replacing the bridge function by a bridge functional of the reference system - it was found out that this method is able to give very accurate results for the structure and thermodynamics of a large variety of one-component and binary liquid systems. ${ }^{13}$ Therefore, with replacement of the MD simulations by the MHNC method we can obtain accurate results for the ion-ion and electron-ion RDF's for dense fluids via the QHNC method using the Rosenfeld bridge functional.

In a plasma, density and temperature vary over a wide range. Therefore, in order to check the applicability of the approach presented here to a strongly coupled plasma (where no reliable experimental data for particle correlations are available), it is important to examine to what extent the QHNC equations can describe a liquid metal in a wide range of densities and temperatures. Recently, using high-intensity $\mathrm{x}$ rays of synchrotron radiation Tsuji et al. ${ }^{14}$ measured the structure factors of liquid $\mathrm{Rb}$ at high pressures: $0.2,2.5,3.9$, 
and $6.1 \mathrm{GPa}$. These pressure values bring along a compression of liquid $\mathrm{Rb}$ : the corresponding density values are estimated to be $1.07,1.41,1.56$, and 1.95 times the normal density; that is, $r_{\mathrm{s}}=5.27,4.81,4.65$, and 4.31 in terms of the Wigner-Seitz radius $a=r_{\mathrm{s}} a_{\mathrm{B}} \quad\left(a_{\mathrm{B}}\right.$ : Bohr's radius), respectively. ${ }^{15}$ Tsuji et al. observed that liquid $\mathrm{Rb}$ is uniformly compressed with increasing pressure. In this context it should be mentioned that Shimojo et al. ${ }^{16}$ performed firstprinciples molecular-dynamics simulation for liquid $\mathrm{Rb}$ under pressure $(0,2.5$, and $6.1 \mathrm{GPa})$ and compared their results with the RDF's extracted from the structure factors measured by Tsuji et al.: they found that liquid $\mathrm{Rb}$ is compressed uniformly at $2.5 \mathrm{GPa}$, but that some deviation from uniform compression is observed at $6.1 \mathrm{GPa}$. In the present work, using the QHNC method in combination with the Rosenfeld bridge functional for the reference hard-sphere system we have calculated the structure factors of compressed rubidium, the pressure ranging from 0 to $6.1 \mathrm{GPa}$, and compared these data with the experimental results. We find reasonable agreement with these experimental data, although in two cases the density has to be slightly readjusted. In this context it must be mentioned that experimental results obtained under these extreme conditions bear some uncertainty: Tsuji ${ }^{17}$ reports that the estimated densities and the height of the main peak in the static structure factor are affected with errors, and that the low- $Q$ behavior of the $6.1 \mathrm{GPa}$ state is rather uncertain, while the positions of the main peaks in the $S(Q)$ are very accurate. We confirm with our results the uniform compression model. We find out that the effective ion-ion potentials are insensitive to this pressure variation, a feature which is obviously typical for liquid alkali metals (we also confirm these observations for the case of liquid lithium). We finally observe that the structure factors coincide in one single curve if $Q$ is scaled in units of the Wigner-Seitz radius $a$.

The paper is organized as follows: in the subsequent section we briefly outline the QHNC method and give a few details about the Rosenfeld bridge functional. In Sec. III we discuss our results and compare them with experimental data. The paper is concluded by a summary.

\section{QHNC THEORY AND THE BRIDGE FUNCTIONAL}

In the present section, we give a brief outline of the QHNC theory and the integration of the Rosenfeld bridge functional in an integral equation approach for a onecomponent fluid.

Let us consider a liquid metal or a plasma as a mixture of electrons and ions interacting through pair potentials $v_{i j}(r)$ $[i, j=\mathrm{e}$ or $\mathrm{I}]$. In this mixture, the ion-ion and electron-ion RDF's $g_{i I}(r)$ are identical with the ion- and electron-density distributions under the external potential caused by a fixed ion at the origin respectively, since the ions behave as a classical fluid in a liquid metal. ${ }^{2}$ In general, DF theory enables us to provide exact expressions for the density distributions in an inhomogeneous system caused by an external potential. Hence, by applying DF theory to this mixture with densities $n_{0}^{i}$, we can derive exact expressions for the ion-ion and electron-ion RDF's in terms of direct correlation functions (DCF's) $C_{i j}(r)$ and bridge functions $B_{i \mathrm{I}}(r)$ as follows: ${ }^{2}$

$$
g_{\mathrm{II}}(r)=\exp \left[-\beta U_{\mathrm{I}}^{\mathrm{eff}}(r)\right],
$$

$$
g_{\mathrm{eI}}(r)=n_{\mathrm{e}}^{0 f}\left(r \mid U_{\mathrm{e}}^{\mathrm{eff}}\right) / n_{0}^{e},
$$

with

$$
\begin{aligned}
U_{i}^{\mathrm{eff}}(r) \equiv & v_{i \mathrm{I}}(r)-\frac{1}{\beta}\left[\sum_{l} \int C_{i l}\left(\left|\mathbf{r}-\mathbf{r}^{\prime}\right|\right)\right. \\
& \left.\times n_{0}^{l}\left[g_{l \mathrm{I}}(r)-1\right] d \mathbf{r}^{\prime}+B_{i \mathrm{I}}(r)\right] \\
= & v_{i \mathrm{I}}(r)-\Gamma_{i \mathrm{I}}(r) / \beta-B_{i \mathrm{I}}(r) / \beta .
\end{aligned}
$$

The wave equation for an electron under the external potential $U_{\mathrm{e}}^{\text {eff }}(r)$ is solved to provide the total electron-density distribution $n_{\mathrm{e}}(r)$ around a nucleus, which is divided into the bound-electron and free-electron parts: $n_{\mathrm{e}}\left(r \mid U_{\mathrm{e}}^{\text {eff }}\right)$ $\equiv n_{\mathrm{e}}^{0 b}\left(r \mid U_{\mathrm{e}}^{\mathrm{eff}}\right)+n_{\mathrm{e}}^{0 f}\left(r \mid U_{\mathrm{e}}^{\mathrm{eff}}\right)$ by the criterion whether their eigenfunctions belong to bound or free levels. In Eq. (2.2), the free-electron part of the density distribution, $n_{\mathrm{e}}^{0 f}\left(r \mid U_{\mathrm{e}}^{\mathrm{eff}}\right) / n_{0}^{e}$, becomes identical to the electron-ion RDF; the bound-electron part $n_{\mathrm{e}}^{0 b}\left(r \mid U_{\mathrm{e}}^{\text {eff }}\right)$ is taken to form an ion and contributes to generate the electron-ion interaction $v_{\mathrm{eI}}(r)$. These expressions for $g_{i \mathrm{I}}(r)$ can be rewritten in the form of a set of integral equations for a one-component fluid. $^{2}$ One of them is a usual integral equation for the DCF $C(r)$ of a one-component fluid:

$$
C(r)=\exp \left[-\beta v_{\mathrm{eff}}(r)+\gamma(r)+B_{\mathrm{II}}(r)\right]-1-\gamma(r),
$$

with an interaction $v_{\text {eff }}(r)$ defined by

$$
\beta v_{\text {eff }}(Q) \equiv \beta v_{\text {II }}(Q)-\frac{\left|C_{\text {eI }}(Q)\right|^{2} n_{0}^{\mathrm{e}} \chi_{Q}^{0}}{1-n_{0}^{\mathrm{e}} C_{\text {ee }}(Q) \chi_{Q}^{0}} ;
$$

the other is an equation for the effective interaction $v_{\text {eff }}(r)$, that is expressed in the form of an integral equation for the electron-ion DCF $C_{\mathrm{eI}}(r)$ :

$$
\hat{B} C_{\mathrm{eI}}(r)=n_{\mathrm{e}}^{0 f}\left(r \mid v_{\mathrm{eI}}-\Gamma_{\mathrm{eI}} / \beta-B_{\mathrm{eI}} / \beta\right) / n_{0}^{\mathrm{e}}-1-\hat{B} \Gamma_{\mathrm{eI}}(r) .
$$

In these equations, $\chi_{Q}^{0}$ is the density response function of the noninteracting electrons with an electron density $n_{0}^{\mathrm{e}}$ and $\gamma(r) \equiv \int C\left(\left|\mathbf{r}-\mathbf{r}^{\prime}\right|\right) n_{0}^{\mathrm{I}}\left[g_{\mathrm{II}}\left(r^{\prime}\right)-1\right] d \mathbf{r}^{\prime}$. Furthermore, $\hat{B}$ denotes an operator defined by

$$
\mathcal{F}_{\mathbf{Q}}[\hat{B} f(r)] \equiv \chi_{Q}^{0} \int e^{i \mathbf{Q} \cdot \mathbf{r}} f(r) d \mathbf{r} .
$$

It should be kept in mind that the electron-ion DCF in Eq. (2.6) plays the role of a nonlinear pseudopotential, which takes into account nonlinear electron accumulations around an ion and the influence of other ions in the form of a linearresponse expression; if the electron-ion DCF $C_{\mathrm{eI}}(r)$ is replaced by a usual pseudopotential $-\beta w_{\mathrm{b}}(r)$, then $v_{\text {eff }}(Q)$ in Eq. (2.6) becomes an effective ion-ion interaction in pseudopotential theory based on the linear-response formalism.

From the above formal and exact expressions (2.5)-(2.7), the QHNC equations are obtained by introducing the following five approximations. ${ }^{2}$

(1) The electron-ion bridge function is neglected in Eq. (2.7): $B_{\mathrm{eI}} \simeq 0$ (the HNC approximation). 
(2) The bridge function $B_{\text {II }}$ of the ion-electron mixture is approximated by that of a one-component hard-sphere fluid [modified HNC (MHNC) approximation ${ }^{18}$ ].

(3) The electron-electron DCF $C_{\mathrm{ee}}(Q)$ in the ion-electron mixture is approximated by that of the jellium model: $C_{\text {ee }}(Q) \simeq-\beta v_{\text {ee }}(Q)\left[1-G^{\mathrm{jell}}(Q)\right]$, which is written in terms of the the local-field correction (LFC) $G^{\text {jell }}(Q)$. In our calculation we use the LFC proposed by Geldart and Vosko. ${ }^{19}$

(4) An approximate $v_{\mathrm{eI}}(r)$ is obtained by treating a liquid metal as a nucleus-electron mixture ${ }^{20}$ in the form:

$$
\begin{aligned}
v_{\mathrm{eI}}(r)= & -Z_{\mathrm{A}} \frac{e^{2}}{r}+\int v_{\mathrm{ee}}\left(\left|\mathbf{r}-\mathbf{r}^{\prime}\right|\right) n_{\mathrm{e}}^{\mathrm{b}}\left(r^{\prime}\right) d \mathbf{r}^{\prime} \\
& +\mu_{\mathrm{XC}}\left[n_{\mathrm{e}}^{\mathrm{b}}(r)+n_{0}^{e}\right]-\mu_{\mathrm{XC}}\left(n_{0}^{\mathrm{e}}\right) .
\end{aligned}
$$

Here, $n_{\mathrm{e}}^{\mathrm{b}}(r)$ is the bound-electron distribution and $\mu_{\mathrm{XC}}(n)$ the exchange-correlation potential in the local-density approximation, for which we have adopted the Gunnarsson-Lundqvist ${ }^{21}$ formula. Actually, the boundelectron distribution $n_{\mathrm{e}}^{\mathrm{b}}(r)$ is determined as the boundelectron part of $n_{\mathrm{e}}\left(r \mid U_{\mathrm{e}}^{\text {eff }}\right) \equiv n_{\mathrm{e}}^{0 b}\left(r \mid U_{\mathrm{e}}^{\text {eff }}\right)+n_{\mathrm{e}}^{0 f}\left(r \mid U_{\mathrm{e}}^{\text {eff }}\right)$, when the electron-ion RDF in Eq. (2.7) is calculated from the wave equation for an electron under the external potential $U_{\mathrm{e}}^{\text {eff }}(r)$ $=v_{\mathrm{eI}}(r)-\Gamma_{\mathrm{eI}}(r) / \beta$.

(5) The bare ion-ion interaction is taken as pure Coulombic: $v_{\mathrm{II}}(r)=Z_{\mathrm{I}} e^{2} / r$.

Under these approximations, a set of integral equations (2.5)-(2.7) can be solved; its solution allows the determination of the electron-ion and ion-ion correlations together with the ionization and the electron bound states.

For the MHNC closure relation we have used in this work the parametrization for the bridge function of a suitably chosen hard-sphere reference system that was proposed recently by Rosenfeld. ${ }^{12}$ In this version of the MHNC the universality hypothesis of the bridge function ${ }^{18}$ (which "justifies" the MHNC) is generalized to the level of the bridge functional of the reference system. This functional can be calculated very easily for the general case of an inhomogeneous system of hard spheres ${ }^{22}$ involving only fundamental measures; the functional is then specialized - as required for our case- to a system of homogeneous hard spheres. This fundamental measure bridge functional is given in terms of characteristic quantities of the individual spheres and involves only integrations over known functions. Furthermore, in this approach the functional can be optimized by imposing the test-particle (or source-particle) self-consistency, which is realized by the transition from an inhomogeneous system to a homogeneous one if the source of the external potential becomes a particle of the liquid. ${ }^{23}$ The Ornstein-Zernike equation is then solved for the structure function of the homogeneous system along with the closure relation where the bridge function is calculated by means of the above functional, assuming that the universality hypothesis is valid. The obtained structure function is then fed into the bridge functional yielding an improved bridge function. This procedure is iterated until numerical self-consistency is obtained in a sense that the structure function of the preceding step differs only marginally from the present step.

This method, which we denote by QHNC-MH, is in fact able to produce accurate data for the structure factors of $\mathrm{Rb}$

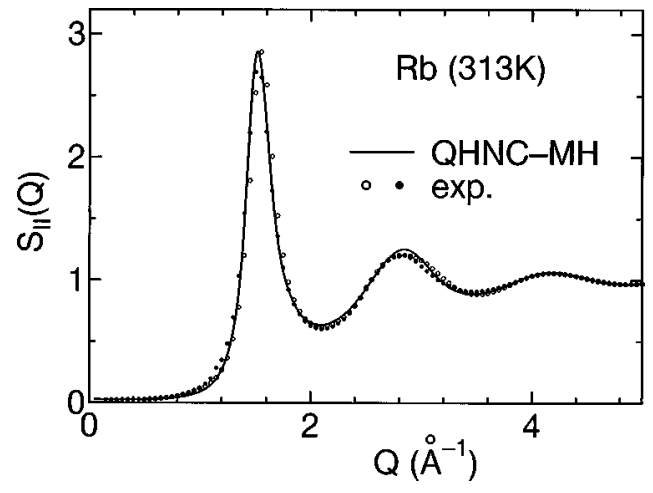

FIG. 1. Ion-ion structure factor $S_{\mathrm{II}}(Q)$ for liquid $\mathrm{Rb}$ at a temperature of $313 \mathrm{~K}$; the QHNC-MH method (in combination with the Rosenfeld bridge functional) yields a structure factor (full curve) in excellent agreement with results observed by the neutron scattering (Ref. 24) (open circles) and x ray (Ref. 25) (full circles).

as demonstrated in Fig. 1 in the direct comparison with experiments at the triple point ( $313 \mathrm{~K}$ and $\left.r_{\mathrm{s}}=5.388\right)$; the results of the neutron-scattering ${ }^{24}$ and $\mathrm{x}$ ray ${ }^{25}$ experiments are denoted by the open and full circles, respectively.

\section{RESULTS AND DISCUSSION}

In this section, we apply the QHNC-MH method to calculate the structure factors for compressed liquid-rubidium under high pressure $(0.2,2.5,3.9$, and $6.1 \mathrm{GPa})$, i.e., exactly the same pressure values under which Tsuji et al. ${ }^{14}$ performed their experiments to measure the structure factors. The corresponding densities are estimated by these authors to be $1.07,1.41,1.56$ and 1.95 times the normal density; the temperatures are $370,520,540$, and $570 \mathrm{~K}$, respectively.

In a first step we apply our method to the $3.9 \mathrm{GPa}$ state and examine how accurately the MHNC equation (in combination with the Rosenfeld bridge function) is able to reproduce the RDF obtained in an MD simulation for the same potential derived by the QHNC-MH method: the comparison is shown in Fig. 2, where the QHNC-MH result (full curve) is found to be undistinguishable from the MD RDF (open circles). This comparison confirms that the QHNC-MH method is able to produce reliable structure data that are as accurate as those obtained in computer experiments even for compressed liquids at high densities; hence, MD simulations

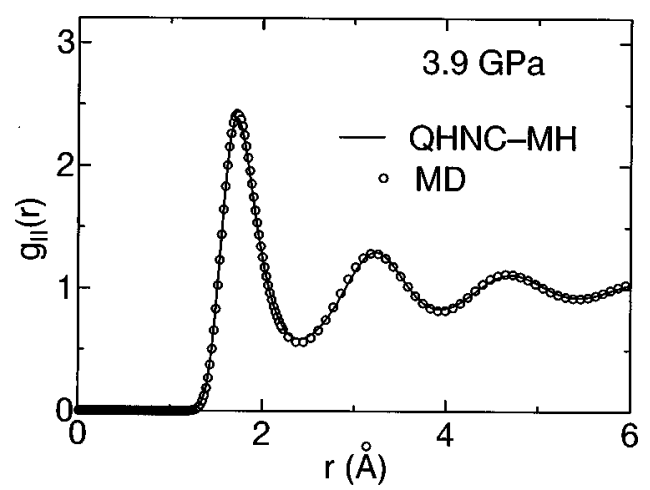

FIG. 2. Ion-ion $\mathrm{RDF} g_{\mathrm{II}}(r)$ at a pressure of $3.9 \mathrm{GPa}$ (full curve) calculated by the QHNC-MH method: results are undistinguishable from those obtained in MD simulations (open circles). 


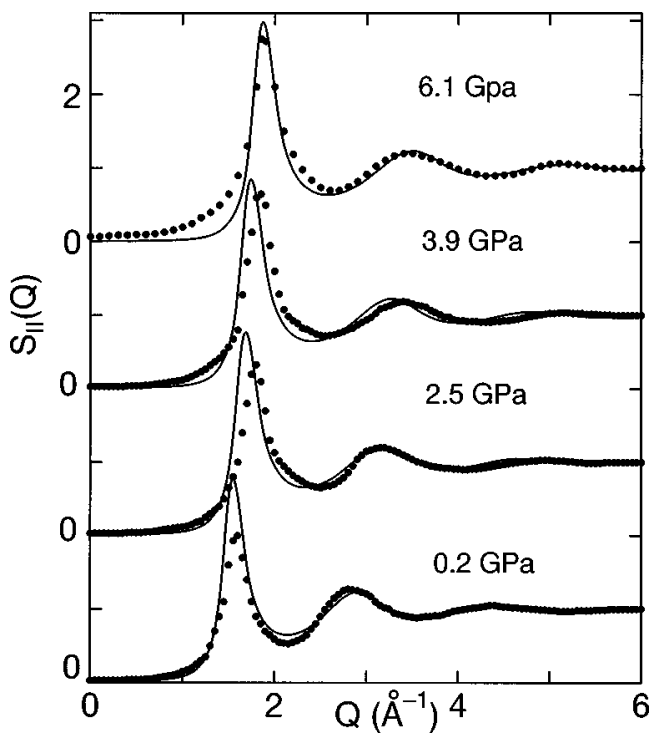

FIG. 3. Structure factors $S_{\mathrm{II}}(Q)$ of liquid $\mathrm{Rb}$ under high pressures: 0.2, 2.5, 3.9, and 6.1 GPa; the QHNC-MH results (full curve) are compared with experiment (Ref. 14) (full circles). The densities corresponding to these pressures are 1.07, 1.41, 1.56, and 1.95 times the normal density $n_{0}$, respectively.

are no longer required in this study.

We can therefore proceed to compare the QHNC-MH results with experimental data for all high-pressure states: the structure factors calculated for pressures ranging from 0.2 to 6.1 GPa are plotted by full curves in Fig. 3 in comparison with the experimental results shown by full circles. We have found that our method gives structure factors in reasonable agreement with experiment for 0.2 and $6.1 \mathrm{GPa}$, in particular in view of the difficulties occurring in experiments under high pressure (cf. discussion in the Introduction). However, in the case of 2.5 and $3.9 \mathrm{GPa}$, the agreement between the theoretical and experimental results is not so convincing: a systematic deviation between the data sets is observed. In their analysis of their experiment, Tsuji et al. ${ }^{14}$ estimated the density of liquid $\mathrm{Rb}$ under pressure from the measured lattice constant of crystalline $\mathrm{Rb}$ including corrections for the thermal expansion and the volume jump at melting. However, according to Tsuji, ${ }^{17}$ there remains an uncertainty in the evaluation of the density that may be responsible for these

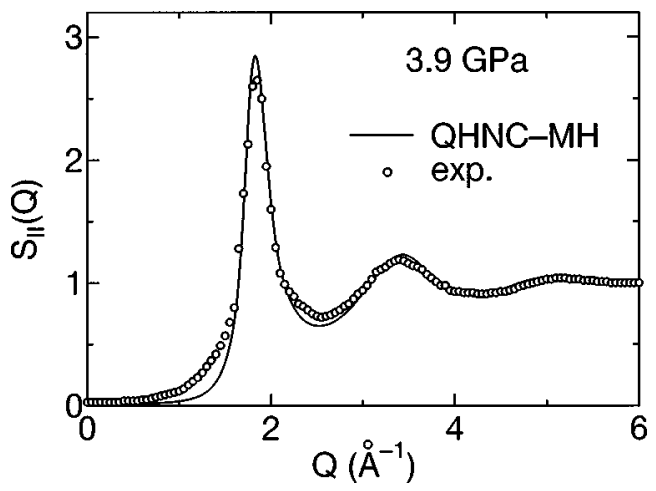

FIG. 4. The reestimated structure factor $S_{\mathrm{II}}(Q)$ (full curve) of liquid $\mathrm{Rb}$ at $3.9 \mathrm{GPa}$ where the Wigner-Seitz radius $a$ has been decreased by a factor of $1 / 1.05$ as a unit of length; reasonable agreement with experimental results (Ref. 14) (open circles) is observed.

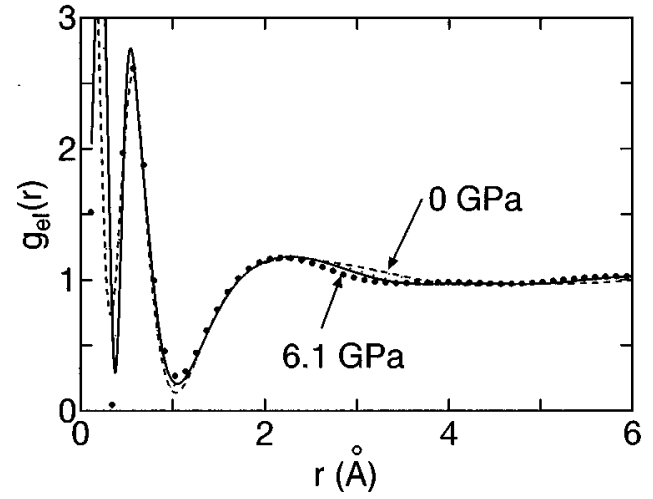

FIG. 5. The electron-ion RDF's $g_{\mathrm{eI}}(r)$ of liquid Rb under pressure (ranging from 0 to $6.1 \mathrm{GPa}$ ); note that the $g_{\text {el }}$ 's remain almost unchanged under pressure variation.

deviations. Therefore, we have modified the density for the 3.9 GPa case: when decreasing the ion-sphere radius (Wigner-Seitz radius) $a\left[a=(3 V / 4 \pi)^{1 / 3}\right.$ and $V$ being the specific volume per ion] by a factor of $1 / 1.05$ we can find indeed a reasonable agreement between the theoretical and experimental results for the $3.9 \mathrm{GPa}$ state, as shown in Fig. 4 ; a similar agreement is found also for the $2.5 \mathrm{GPa}$ case if the Wigner-Seitz radius $a$ is decreased by $5 \%$.

It should be mentioned that the concept of our QHNC method is for high pressures as reliable and valid as for the room pressure, since all the approximations entering this method remain valid as the pressure is increased. This fact can be seen from the result for the electronic structure, which will be discussed in the following. The bound levels of the ion in compressed liquid $\mathrm{Rb}$ are almost the same as those at room pressure. As a consequence, the electron-ion RDF's remain-as displayed in Fig. 5-almost unchanged for the five states considered. The electron-ion DCF $C_{\mathrm{eI}}(r)$ is determined by Eq. (2.7). Figure 6 illustrates the pressure variation of the electron-ion DCF $C_{\mathrm{eI}}(r)$, which-as noted aboveplays the role of a nonlinear pseudopotential in the expression for the effective ion-ion interaction [cf. Eq. (2.5)]; also the electron-ion DCF does not change significantly under these pressure variations. For liquid alkalis, it is well known that the Ashcroft pseudopotential provides effective pair po-

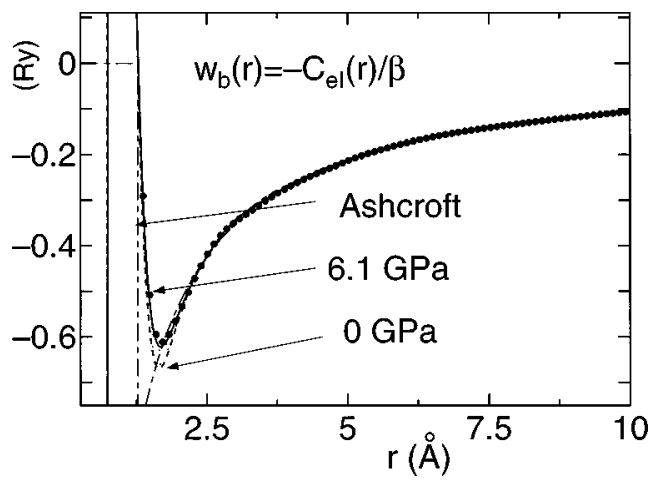

FIG. 6. The electron-ion DCF's $C_{\mathrm{eI}}(r)$ of liquid Rb under pressure (ranging from 0 to $6.1 \mathrm{GPa}$ ); these functions play the role of a nonlinear pseudopotential $w_{\mathrm{b}}(r)$ to determine the effective ion-ion interaction. The dash-dotted curve denotes the Ashcroft pseudopotential with $r_{\mathrm{c}}=1.27 \AA$. 
tentials which produce with standard liquid state theories RDF's in good agreement with experiments, if the core radius $r_{\mathrm{c}}$ is properly adjusted. In Fig. 6, we plotted the Ashcroft pseudopotential with the core radius $r_{\mathrm{c}}=2.40 a_{\mathrm{B}}$ $=1.27 \AA$, which produces for the state $\left(T=313 \mathrm{~K}, r_{s}\right.$ $=5.388$ ) practically the same structure factor as obtained by the QHNC-MH method (cf. Fig. 1) using the Gerdart-Vosko LFC. It is interesting that-although the Ashcroft pseudopotential is quite different from the QHNC pseudopotential at room pressure - these two pseudopotentials produce an almost identical structure factor.

Here, note that the electron-ion structure factor $S_{\mathrm{eI}}(Q)$, the Fourier transform of the electron-ion RDF, is written in the following form:

$$
\begin{aligned}
S_{\mathrm{eI}}(Q) & =\sqrt{n_{0}^{\mathrm{I}} n_{0}^{\mathrm{e}}} C_{\mathrm{eI}}(Q) \chi_{Q}^{0} / D(Q) \\
& =\frac{\rho(Q)}{\sqrt{Z_{\mathrm{I}}}} S_{\mathrm{II}}(Q),
\end{aligned}
$$

where

$$
\begin{gathered}
\rho(Q) \equiv \frac{n_{0}^{\mathrm{e}} C_{\mathrm{eI}}(Q) \chi_{Q}^{0}}{1-n_{0}^{\mathrm{e}} C_{\mathrm{ee}}(Q) \chi_{Q}^{0},} \\
D(Q) \equiv\left[1-n_{0}^{\mathrm{I}} C_{\mathrm{II}}(Q)\right]\left[1-n_{0}^{\mathrm{e}} C_{\mathrm{ee}}(Q) \chi_{Q}^{0}\right] \\
-n_{0}^{\mathrm{I}} n_{0}^{\mathrm{e}}\left|C_{\mathrm{eI}}(Q)\right|^{2} \chi_{Q}^{0} .
\end{gathered}
$$

Hence, Eq. (3.2) can be represented in $r$ space as

$$
n_{0}^{\mathrm{e}} g_{\mathrm{eI}}(r)=\rho(r)+n_{0}^{\mathrm{I}} \int \rho\left(\left|\mathbf{r}-\mathbf{r}^{\prime}\right|\right) g_{\mathrm{II}}\left(r^{\prime}\right) \mathbf{r}^{\prime},
$$

which states that the free-electron distribution $n_{0}^{\mathrm{e}} g_{\text {eI }}(r)$ around an ion can be described exactly by the superposition of surrounding " "neutral pseudoatoms." Each ion carries a screening electron-cloud $\rho(r)$ [with $\int \rho(r) d \mathbf{r}=Z_{\mathrm{I}}$ ], and makes it thus electrically neutral (including the core electrons) as if it were an atom. Therefore, in this formalism a liquid metal can be considered to be composed by neutral pseudoatoms. Using Eq. (3.3), the free-electron density distribution of a pseudoatom is calculated for liquid $\mathrm{Rb}$ under high pressures (cf. Fig. 7): the results indicate that the electron-density distribution of a pseudoatom suffers no significant change outside of the core region (the region within about $2 \AA$ where the bound-electron density is large) under this pressure variation. The dash-dotted curve in this figure denotes the electron-density distribution of a pseudoatom determined via the Ashcroft pseudopotential, which does not produce the inner-core structure. The inner part of the density distribution does not contribute to the ion-ion effective interaction significantly; the outer part (from around $2 \AA$ onwards), where the bound-electron density becomes negligible, may be important to determine the ion-ion interaction. Summarizing, we can conclude that a pseudoatom including bound electrons remains almost unchanged in comparison to room pressure even when the density is increased by a factor of nearly 2 (high pressure state $6.1 \mathrm{GPa}$ ). On the other hand, Tsuji et al. expected that a hard-sphere mode ${ }^{26}$ might be successfully applied to describe the structure of liquid alkali metals and that the effective diameter of the hard sphere

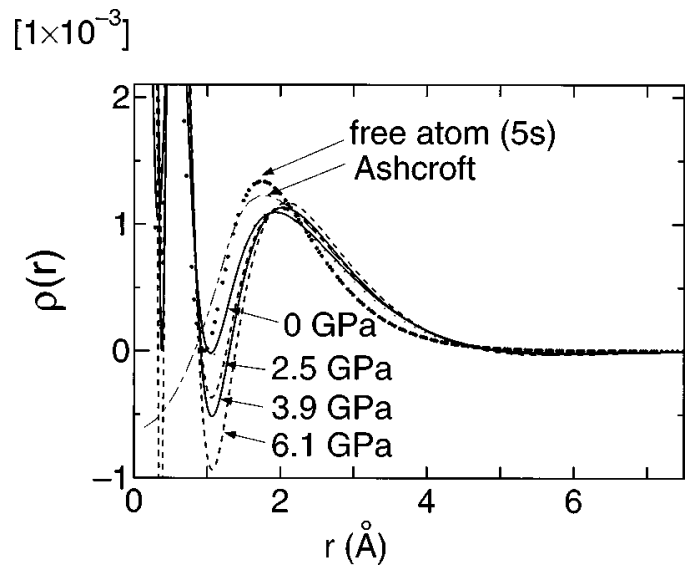

FIG. 7. The electron-density distribution $\rho(r)$ of a pseudoatom in liquid $\mathrm{Rb}$ under pressure (ranging from 0 to $6.1 \mathrm{GPa}$ ); the $5 s$-electron density distribution of a free atom is displayed for comparison (small full circles). $\rho(r)$ is plotted in units of $a_{\mathrm{B}}^{-3}$ ( $a_{\mathrm{B}}$ being the Bohr radius). The dash-dotted curve indicates the density distribution calculated with use of the Ashcroft pseudopotential, which cannot describe an inner-core structure due to the pseudization.

should vary with pressure due to the change in the screening effect; that is, the size of a pseudoatom is assumed to be changed with pressure. Based on this model, the effective hard-sphere radius $\sigma_{\mathrm{H}}$ is considered to vary under pressure variation keeping the packing fraction $\eta=\pi n_{0}^{\mathrm{I}} \sigma_{\mathrm{H}}^{3} / 6$ to be constant at a value of 0.45 ; this leads to a uniformcompression model in which the position of first peak $Q_{1}$ in the structure factor should increase proportionally to $\left(n_{0}^{\mathrm{I}}\right)^{1 / 3}$ with increasing pressure.

We also find in our approach that the effective ion-ion interaction for liquid $\mathrm{Rb}$ is practically invariant under these pressure variations (cf. Fig. 8). It remains almost the same as the potential at room pressure, in particular, in the region where the first peak of the ion-ion RDF appears under these high pressures: the large black circle in Fig. 8 denotes the first peak position $(3.89 \AA)$ of the ion-ion RDF at the pressure $6.1 \mathrm{GPa}$. It should be noted that the effective ion-ion

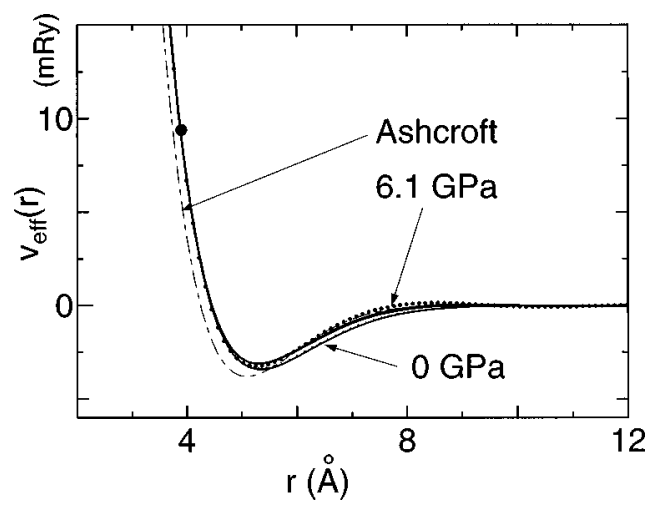

FIG. 8. The effective ion-ion interaction $v_{\text {eff }}(r)$ of liquid $\mathrm{Rb}$ under pressure (ranging from 0 to $6.1 \mathrm{GPa}$ ); the effective interaction for compressed liquid $\mathrm{Rb}$ remains practically unchanged with respect to room pressure. The large black circle denotes the first peak position of the ion-ion RDF at pressure $6.1 \mathrm{GPa}$; the potential in the vicinity of this point contributes mainly to the structure factor. The Ashcroft potential is plotted by the thin dash-dotted curve. 


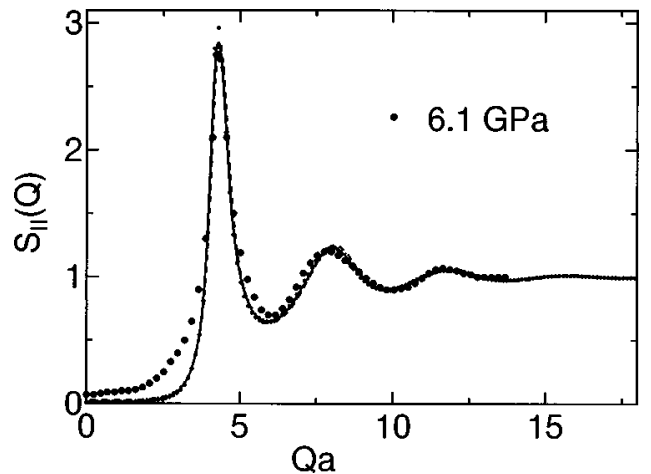

FIG. 9. Structure factors $S_{\mathrm{II}}(Q)$ of liquid $\mathrm{Rb}$ calculated for 0 , $0.2,2.5,3.9$, and $6.1 \mathrm{GPa}$; all results are scaled in units of the Wigner-Seitz radius $a$ and practically coincide in one curve; the experimental result (Ref. 14) for 6.1 GPa is plotted by full circles.

potential (dash-dotted curve) determined from the Ashcroft pseudopotential has almost the same gradient in the repulsive region as the potential obtained from the QHNC-MH method. In contrast to the hard-sphere model, where the essential repulsive part of the effective ion-ion potential should be scaled in units of $a$, no scaling feature is observed for the effective ion-ion interaction in units of the Wigner-Seitz radius $a$. Nevertheless, all the calculated structure factors for all these five pressure values (with densities estimated by Tsuji et al. ${ }^{14}$ ) almost coincide in one single curve when scaling the wave number in units of the Wigner-Seitz radius $a$ (as shown in Fig. 9). In this figure, a deviation between calculated and experimental results is in the low $Q$ region. We know from Tsuji ${ }^{17}$ that the experimental values in this region are affected with large error bars and we expect that these deviations between theoretical and experimental results will disappear when more refined experimental data are available. In this context, it should be noticed that this scaling property can be ascribed generally to a specific feature characteristic for ion-ion potentials of alkali liquids as will be discussed below.

Even if the liquid-rubidium potential under these pressure variations can be considered as invariant with respect to the one at room pressure (neglecting small deviation), we have also observed the above mentioned scaling property in the structure factor (cf. Fig. 9); this means that the liquidrubidium potential has a very special feature. For a general liquid (the Lennard-Jones potential for example), it is impossible to display the structure factor of two different states, scaled in length units in such a way that they practically coincide. For a liquid alkali metal such a scaling property can be observed: to examine this, let us consider liquid $\mathrm{Li}$ as a further example. At first we calculate the effective ion-ion interaction of liquid $\mathrm{Li}$ at room pressure $\left(470 \mathrm{~K}, n_{0}\right.$ with $r_{\mathrm{s}}$ $=3.308$ ), and assume - as for $\mathrm{Rb}$ - that the effective Li potential is unchanged with respect to the one at room pressure. Then, we can easily find the following three sets of temperatures and densities: $\left(470 \mathrm{~K}, n_{0}\right),\left(600 \mathrm{~K}, 1.34 n_{0}\right)$, and $(750$ $\mathrm{K}, 2.37 n_{0}$ ), i.e. states for which the structure factors can be scaled almost in a single curve in units of $a$, as demonstrated in Fig. 10; here, open circles denote the result of the neutronscattering experiment ${ }^{27}$ at room pressure. The above set of densities and temperatures have been chosen just because these states exhibit a clear scaling. As mentioned before, the

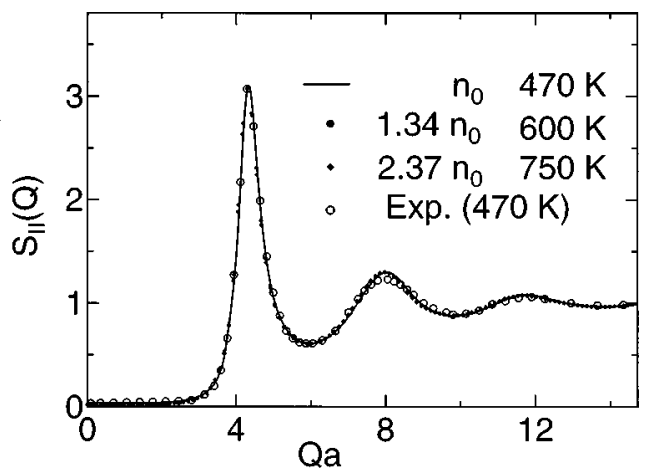

FIG. 10. Structure factors $S_{\mathrm{II}}(Q)$ of liquid Li calculated for two states, $\left(1.34 n_{0}, 600 \mathrm{~K}\right)$ and $\left(2.34 n_{0}, 750 \mathrm{~K}\right)$, under the assumption that the effective ion-ion interaction is the same as that for room pressure. The structure factor (full curve) of liquid Li at room pressure is also plotted for comparison; when scaled in units of the Wigner-Seitz radius $a$, all three structure factors practically coincide in a single curve. The experimental data (Ref. 27) (neutron scattering) at room pressure is plotted by open circles.

ion-ion potentials based on both Ashcroft and the QHNC-MH pseudopotentials yield an almost identical structure factor. However, the Ashcroft and QHNC-MH pseudopotentials are quite different from each other as is shown in Fig. 8; nevertheless, it is confirmed that this Ashcroft ion-ion potential has also the same scaling property for the same states as examined by the QHNC-MH method. This fact can be interpreted that the structure factor is determined mainly by the repulsive part of the ion-ion interaction where both potentials have almost the same gradient as was shown in Fig. 8. For alkali liquid metals, this scaled structure factor is not very sensitive to states specified by the plasma parameter $\Gamma \equiv \beta e^{2} / a$ and $r_{s} \equiv a / a_{\mathrm{B}}$ in the sufficiently high-density region. This is the main reason why structure factors of all alkali liquids (from $\mathrm{Li}$ to $\mathrm{Cs}$ ) near the triple point coincide almost in a single curve when scaling $Q$ in units of the Wigner-Seitz radius $a$, as was observed experimentally ${ }^{28,29}$ and theoretically. ${ }^{30,11,31}$

This scaling property in the structure factors of liquid $\mathrm{Rb}$ signifies that the first peak of the structure factor appears almost at the same position $Q_{1} a$ (in scaled units) for all these pressures; this means that the position of the first peak $Q_{1}$ in the structure factor (taken in absolute values) should increase proportionally to $x=\left(V / V_{0}\right)^{-1 / 3}$, where $V_{0}$ is the specific volume at room pressure. In our calculation, this peak position in reduced units is estimated from Fig. 9 to be $Q_{1} a$ $=4.30$, from which we obtain the relation $Q_{1}=1.51 x$ because of $a=r_{\mathrm{s}} a_{\mathrm{B}}=2.58 / x$; this linear relation is plotted in Fig. 11. In this figure, the full and the open circles denote the experimental points obtained for several states by Tsuji et al. $;^{32}$ in particular, the five full circles represent those states which we have investigated in our theoretical study, i.e., for pressure values $0,0.2,2.5,3.9$, and $6.1 \mathrm{GPa}$, respectively. These experimental $Q_{1}$ points in Fig. 2.7 are close to the linear relation which was determined from the calculated structure factors; thus, this figure demonstrates that the uniform-compression model (corresponding to a linear relation denoted by the full line) is indeed valid, although there is some uncertainty due to difficulties in estimating the density and to problems in experiments under high pressure, in general. 


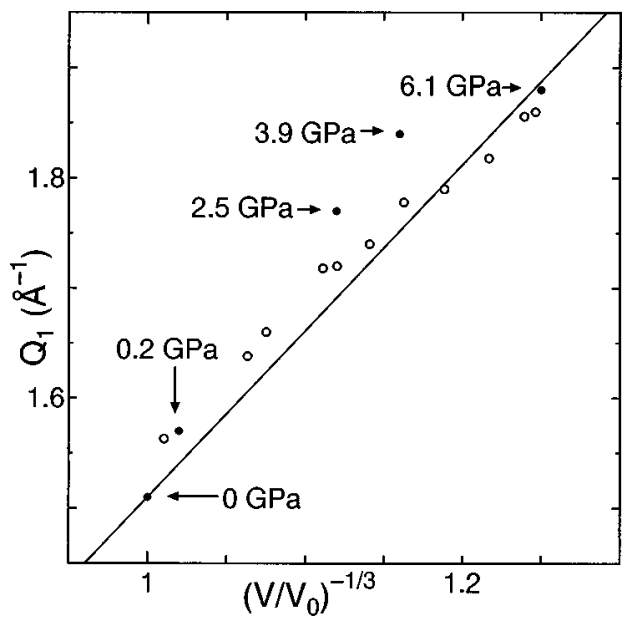

FIG. 11. The position of the first peak $Q_{1}$ in the structure factor for liquid $\mathrm{Rb}$ as a function of $\left(V / V_{0}\right)^{-1 / 3}$; open and full circles represent experimental results (Ref. 32). In particular, the full circles are the points determined from the experimental structure factors under pressure: 0, 0.2, 2.5, 3.9, and 6.1 GPa plotted in Figs. 1 and 3. The solid line denotes our calculated results (uniform compression model) derived from the first peak position $\left(Q_{1} a=4.30\right)$ in the scaled structure factor in Fig. 9.

\section{CONCLUSIVE DISCUSSION}

The QHNC method in combination with the Rosenfeld bridge functional has been shown to reproduce the experimental structure factors of liquid $\mathrm{Rb}$ under high pressures (ranging from 0 to $6.1 \mathrm{GPa}$ ) in general with a reasonable, sometimes even with a rather good accuracy; this has to be seen in particular in view of the experimental uncertainties (peak height, density values, low- $Q$ behavior) which are caused by the extreme experimental conditions. For the case of 2.5 and $3.6 \mathrm{GPa}$ the experimentally estimated densities have to be readjusted in terms of a $5 \%$ variation of the Wigner Seitz radius $a$. Furthermore we observe that the structure factors coincide almost in one single curve if wave numbers are scaled in units of $a$ (Fig. 9). This indicates clearly that liquid $\mathrm{Rb}$ is uniformly compressed as the pressure is increased: this, in turn, signifies that the first peak position $Q_{1}$ of the structure factors increases proportional to $\left(V / V_{0}\right)^{-1 / 3}$ (Fig. 11).

It must be mentioned that in contrast to our result, Shimojo et al. ${ }^{16}$ conclude from their result obtained in the firstprinciples MD simulations that some deviation from uniform compression exists for the $6.1 \mathrm{GPa}$ state, though liquid $\mathrm{Rb}$ is compressed uniformly at $2.5 \mathrm{GPa}$. Their experimental RDF's for 2.5 and $6.1 \mathrm{GPa}$ are obtained by a Fourier transform of the experimental structure factors of Tsuji et al. On the basis of the RDF's they observe a different behavior than on the basis of the structure factor, although they have used the same experimental structure factors and $\mathrm{Rb}$ states as we did: (i) the first peak position in the RDF at $2.5 \mathrm{GPa}$ follows the uniform compression model, while the first peak position in the RDF at 6.1 GPa shows a deviation from the uniform compression model. Thus they have asserted that their calculated result agrees with the experiment. (ii) In contrast to their RDF's, the experimental structure factor at $2.5 \mathrm{GPa}$ shows a substantial deviation from the uniform compression compared to the $6.1 \mathrm{GPa}$ state; the position $Q_{1}$ of the first peak in $S_{\mathrm{II}}(Q)$ lies far away from the uniform compression line in Fig. 11 while, on the other hand, the experimental structure factor at $6.1 \mathrm{GPa}$ shows that the uniform compression model is still valid: the $Q_{1}$ point for $6.1 \mathrm{GP}$ in Fig. 11 is very close to the uniform compression line.

According to Tsuji, ${ }^{17}$ this discrepancy between the conclusions based on the RDF's and the structure factors is possible, since there is some experimental ambiguity in the value of the peak height in the structure factor while the peak position is accurate and reliable. Nevertheless, from all the experimental data displayed in Fig. 11, we can conclude that liquid $\mathrm{Rb}$ is compressed uniformly up to a pressure of 6.1 $\mathrm{GPa}$; however, a more detailed discussion if deviations from the uniform compression model (cf. Fig. 11) have any physical meaning is not very conclusive, due to the uncertainty in the evaluation of the experimental density and the difficulties encountered in experiments under high pressure.

It is interesting to notice that the structure factors of compressed liquid $\mathrm{Rb}$ coincide practically in one single curve if wave numbers are scaled in units of the Wigner-Seitz radius $a$, despite the fact that the effective ion-ion interaction remains under pressure unchanged with respect to room pressure; this means that interaction potentials of liquid alkali metals have a special characteristic property, as we have demonstrated and confirmed in addition for the case of liquid $\mathrm{Li}$ in Fig. 10. The neutral pseudoatom in compressed liquid $\mathrm{Rb}$ remains almost unchanged under pressure variation, which, in turn, is the reason why the effective ion-ion interaction remains practically invariant, similar to stateindependent interactions, such as the one for liquid argon.

We have demonstrated in this contribution that the QHNC method can treat the "outer-structure", problem (i.e., calculation of the ion-ion and electron-ion RDF's) and the "innerstructure", problem (i.e., calculation of the electronic structure of the ion) in a self-consistent way using the atomic number as the only input data: therefore, this method can be considered to be very useful to treat a plasma, where the ionization $Z_{\mathrm{I}}$ is not known beforehand and where there is no way of constructing a pseudopotential to give the effective ion-ion interaction. From the successful results for compressed liquid $\mathrm{Rb}$ presented here we can conclude that the QHNC method is expected to be nicely applicable for plasma states in a wide range of densities and temperatures.

\section{ACKNOWLEDGMENTS}

We thank Professor Tsuji and Dr. Morimoto for sending us numerical experimental data and for discussions on the analysis of the experimental data. G.K. acknowledges financial support by the Österreichische Forschungsfonds under Project No. P11194-PHY. 
${ }^{1}$ J. Chihara, Phys. Rev. A 33, 2575 (1986).

${ }^{2}$ J. Chihara, J. Phys.: Condens. Matter 3, 8715 (1991).

${ }^{3}$ J. Chihara, Phys. Rev. A 40, 4507 (1989).

${ }^{4}$ M. Ishitobi and J. Chihara, J. Phys.: Condens. Matter 4, 3679 (1992).

${ }^{5}$ M. Ishitobi and J. Chihara, J. Phys.: Condens. Matter 5, 4315 (1993).

${ }^{6}$ J. Chihara and S. Kambayashi, J. Phys.: Condens. Matter 6, 10221 (1994).

${ }^{7}$ F. Perrot, Phys. Rev. E 42, 4871 (1990).

${ }^{8}$ L. E. González, D. J. González, and K. Hoshino, J. Phys.: Condens. Matter 5, 9261 (1993).

${ }^{9}$ L. E. González, A. Meyer, M. P. Inigues, D. J. González, and M. Silbert, Phys. Rev. E 47, 4120 (1993).

${ }^{10}$ J. Chihara, Phys. Rev. E 44, 1247 (1991).

${ }^{11}$ S. Kambayashi and J. Chihara, Phys. Rev. E 53, 6253 (1996).

${ }^{12}$ Y. Rosenfeld, J. Chem. Phys. 98, 8126 (1993).

${ }^{13}$ G. Kahl, B. Bildstein, and Y. Rosenfeld, Phys. Rev. E 54, 5391 (1996).

${ }^{14} \mathrm{~K}$. Tsuji, in Elementary Processes in Dense Plasmas, edited by S. Ichimaru and S. Ogata (Addison-Wesley, New York, 1995); K. Tsuji, Y. Katayama, Y. Morimoto, and O. Shimamura, J. NonCryst. Solids 205-207, 295 (1996).

${ }^{15}$ Y. Morimoto (private communication).

${ }^{16}$ F. Shimojo, Y. Zempo, K. Hoshino, and M. Watabe, Phys. Rev. B 55, 5708 (1997).

${ }^{17} \mathrm{~K}$. Tsuji (private communication).

${ }^{18}$ Y. Rosenfeld and N. W. Ashcroft, Phys. Rev. A 20, 1208 (1979).
${ }^{19}$ D. J. W. Geldart and S. H. Vosko, Can. J. Phys. 44, 2137 (1966).

${ }^{20}$ J. Chihara, J. Phys. C 18, 3103 (1985).

${ }^{21}$ O. Gunnarsson and B. I. Lundqvist, Phys. Rev. B 13, 4274 (1976).

${ }^{22}$ Y. Rosenfeld, Phys. Rev. Lett. 63, 980 (1989).

${ }^{23}$ J. K. Percus, Phys. Rev. Lett. 8, 462 (1962); J. K Percus, in The Equilibrium Theory of Classical Fluids, edited by H. L. Frisch and J. L. Lebowitz (Benjamin, New York, 1964), Chap. II, p. 171.

${ }^{24}$ J. R. D. Copley and S. W. Lovesey, in Liquid Metals, 1976, edited by R. Evans and D. A. Greenwood IOP Conf. Proc. No. 30, (The Institute of Physics, Bristol and London, 1977), p. 575.

${ }^{25}$ Y. Waseda, The Structure of Non-Crystalline Materials (McGraw-Hill, New York, 1980).

${ }^{26}$ N. W. Ashcroft and J. Lekner, Phys. Rev. 145, 83 (1966).

${ }^{27}$ H. Olbrich, H. Ruppersberg, and S. Steeb, Z. Naturforsch. A 38A, 1328 (1983).

${ }^{28}$ M. J. Huijben and W. van der Lugt, Acta Crystallogr., Sect. A: Cryst. Phys., Diffr., Theor. Gen. Crystallogr. 35, 431 (1979).

${ }^{29}$ Y. Waseda and K. Suzuki, in Proceedings of the Second International Conference on the Properties of Liquid Metals, edited by S. Takeuchi (Taylor \& Francis, London, 1973), pp. 37.

${ }^{30}$ H. Minoo, C. Deutsh, and J. P. Hansen, J. Phys. (Paris) 38, L-191 (1977).

${ }^{31}$ U. Balucani, A. Torcini, and R. Vallauri, Phys. Rev. B 47, 3011 (1993).

${ }^{32}$ The experimental data for the $x-Q_{1}$ relation are provided by Tsuji in the numerical table. 